ment. Fast drying increases sensitivity and decreases background fog.

$$
\begin{array}{r}
\text { Eastman Kodak Company, } \\
\text { Rochester, N. Y. }
\end{array}
$$

\section{Wagman, N. E. Algol positions from Allegheny photographs.}

The material consists of a series of more than 30 plates taken for parallax from I9I6 to 1923 and a current series of nearly Ioo plates begun in I934 as a part of Burns's program of special objects. Parallax solutions in right ascension and declination have been made for both the old and the new series. The parallax in right ascension as determined by Daniel from the earlier series agrees with that given by the new series. The values of the parallax in declination are likewise in agreement but differ from those in right ascension. However, this difference is considerably less than that found at Sproul. ${ }^{1}$ Com- bining all the Allegheny material we have the relative parallaxes:

$$
\begin{aligned}
\pi_{\alpha} & =+.038 \pm .005 \\
\pi_{\delta} & =+.059 \pm .01 \mathrm{I} \\
\pi_{\alpha+\delta} & =+.04 \mathrm{I} \pm .004
\end{aligned}
$$

The relative proper motion is less than ".oor in both right ascension and declination.

With the above values residuals were computed for all the plates. These were grouped according to phase in the 1.87 year orbit of the third body of the Algol system. Graphs of these residuals in right ascension give evidence of a shift with a half-amplitude of about "o3. Evidence of a smaller shift is seen in declination. Observations are being continued.

I. $A$ p. J. $96,420,1942$.

Allegheny Observatory, Pittsburgh, Pa.

\title{
REPORTS OF OBSERVATORIES, I943-I944
}

Leander McCormick Observatory. University of Virginia, University, Virginia.

Solar motion, galactic rotation and precession. Several solutions have been completed by A. N. Vyssotsky and Emma.T. R. Williams dealing with 78I McCormick proper motion regions north of declination $-25^{\circ}$, together with the proper motions in the Cape Zone between $-40^{\circ}$ and $-52^{\circ}$.

The high accuracies of the individual motions of the stars, which cover seven-eighths of the area of the whole sky, and the great number of stars involved, amounting to twice the number in the General Catalogue, will give results of great importance for three separate problems: (I) the motion of the sun with respect to stars of different magnitudes and different spectral types; (2) the rotation of the galaxy; and (3) the constant of precession of the equinoxes.

To obtain an idea of the possible influence of systematic errors, independent solutions in right ascension and declination were made before combining the whole material into one solution. It was found that the results from right ascension were in satisfactory agreement with those from declination as shown by the following table.

$$
\begin{aligned}
& \text { COMPONENTS OF SOLAR MOTION IN EQUATORIAL } \\
& \text { COORDINATES } \\
& X \quad \text { R.A. Decl. Combined } \\
& X \quad+\text { ".07 } \pm \text { ".03 } \quad+\text { ".04 } \pm \text { ".05 } \quad \text { +..07 } \pm \text { ".03 } \\
& -.98 \pm .03 \quad-\mathrm{r} .00 \pm .05 \quad-.98 \pm .03 \\
& \text { ORRECTIONS TO EQUATORIAL GOMPONENTS OF } \\
& \text { NEWCOMB'S PRECESSION CONSTANTS } \\
& \text { R.A. Decl. Combined }
\end{aligned}
$$

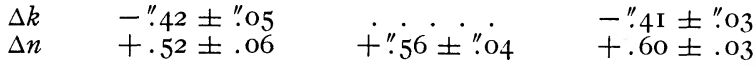

$$
\begin{aligned}
& \text { OORT'S CONSTANTS OF GALACTIC ROTATION } \\
& \text { R.A. Decl. Combined } \\
& \begin{array}{llll}
P & +.63 \pm . .07 & \text { +.".32 } \pm . .05 & +. .43 \pm . .03 \\
Q & -.15 \pm .08 & -.14 \pm .04 & -.17 \pm .03
\end{array}
\end{aligned}
$$

As stated in last year's report, the obser vations have been reduced both to the $\mathrm{FK}_{3}$ system and to that underlying the General Catalogue. It is the consensus of opinion, as expressed in a resolution at the 1938 meeting of the International Astronomical Union, that the $\mathrm{FK}_{3}$ system gives the more reliable results. The McCormick proper motions give abundant verification of this point of view. The values in the table are those referred to the $\mathrm{FK}_{3}$ system.

In order further to substantiate the values of the last four unknowns in the table, the authors took what they considered to be the best parts 